\title{
Effect of Composite Additives on the Zinc Anode of Zinc-Nickel Single-Flow Batteries
}

\author{
Shouguang Yao $^{1, *}$, Dapei Ding ${ }^{1,2}$, Jie Cheng ${ }^{2,3}$, Hao Xu ${ }^{1,3}$, Yusheng Yang ${ }^{2,3}$ \\ ${ }^{1}$ School of Energy and Power Engineering, Jiangsu University of Science and Technology, Zhenjiang, \\ Jiangsu 212003, China \\ ${ }^{2}$ ZhangjiagangSmartgridFanghua electrical energy storage research institute Co., Ltd., Zhangjiagang \\ 215600, China \\ ${ }^{3}$ Chilwee Power Co., Ltd., Huzhou 313100, China \\ *E-mail: zjyaosg@126.com
}

doi: $10.20964 / 2020.11 .58$

Received: 3 May 2020/ Accepted: 13 June 2020 / Published: 30 September 2020

In this study, a nickel-plated steel strip was used as the base material of zinc electrodes. The effect of composite additives, such as $\mathrm{Sn}^{4+}, \mathrm{Ga}^{3+}$, and $\mathrm{Pb}^{2+}$, on zinc deposition and dissolution was tested using cyclic voltammetry, SEM, and constant-current charge/discharge. The cyclic voltammetry results showed that the composite additives could codeposit with zinc and easily form uniform crystal nuclei and dense deposit layers. The Tafel polarization curves demonstrated that the hydrogen evolution reaction on the electrodes in the solution with composite additives was weaker than those of the base solution and a single additive. The constant-current charge/discharge results presented that electrodes in the solution with composite additives obtained high coulombic efficiency but not significantly higher than that of the base solution and a single additive. The SEM results showed the smooth zinc deposit morphology with composite additives and small zinc deposit particles. The results of the selfdischarge test demonstrated that the self-discharge performance of the base solution clearly improved with the addition of composite additives and the residual capacity increased from $49.7 \%$ to $65.6 \%$ after $24 \mathrm{~h}$ in standby state.

Keywords: Zinc-nickel single-flow battery; Composite additives; Charge retention; Coulombic efficiency

\section{FULL TEXT}

(C) 2020 The Authors. Published by ESG (www.electrochemsci.org ). This article is an open access article distributed under the terms and conditions of the Creative Commons Attribution license (http://creativecommons.org/licenses/by/4.0/). 